\title{
Discours de professionnels et discours pour professionnels : le travail collaboratif au service de l'enseignement de l'anglais médical
}

Bridging the gap between medical English and English for medical purposes

through collaborative work

Didier Carnet et Jean-Pierre Charpy

\section{OpenEdition \\ Journals}

Édition électronique

URL : http://journals.openedition.org/asp/4952

DOI : 10.4000/asp.4952

ISSN : 2108-6354

Éditeur

Groupe d'étude et de recherche en anglais de spécialité

Édition imprimée

Date de publication : 1 mars 2017

Pagination : 47-68

ISSN : 1246-8185

Référence électronique

Didier Carnet et Jean-Pierre Charpy, « Discours de professionnels et discours pour professionnels : le travail collaboratif au service de l'enseignement de l'anglais médical », ASp [En ligne], 71 | 2017, mis en ligne le 01 mars 2018, consulté le 10 décembre 2020. URL : http://journals.openedition.org/asp/4952 ; DOI : https://doi.org/10.4000/asp.4952

Ce document a été généré automatiquement le 10 décembre 2020.

Tous droits réservés 


\title{
Discours de professionnels et discours pour professionnels : le travail collaboratif au service de l'enseignement de l'anglais médical
}

\author{
Bridging the gap between medical English and English for medical purposes \\ through collaborative work
}

Didier Carnet et Jean-Pierre Charpy

\section{Introduction}

1 Au sein des langues de spécialité, l'anglais médical constitue un ensemble à portée universelle comportant peu de variantes nationales. Cette universalité repose essentiellement sur un socle terminologique gréco-romain (Faure 2010). Cet ancrage en tant que langue spécialisée se distinguant de la langue générale remonte à l'apparition des premiers dictionnaires dédiés au lexique spécifique de l'anglais médical (Charpy 2011).

2 Dans le contexte de l'enseignement de la langue spécialisée, nous sommes conscients de l'intérêt théorique de ce cadre diachronique pour la formulation d'une définition épistémologique de la spécialité (Van der Yeught 2016) et de la nécessité d'une veille métaphorique et terminologique (Resche 2016). Toutefois, nous proposons d'adopter une perspective plus pragmatique pour présenter et faire le bilan de quatre actions menées à l'UFR Sciences de Santé de Dijon au cours de ces vingt dernières années. Notre objectif est de caractériser les besoins langagiers des professionnels de santé en procédant à une analyse commune des discours spécialisés, réels, fictifs ou didactisés auxquels ils se réfèrent.

3 Cette étude s'inscrit dans le cadre de l'analyse des besoins inspirée de l'approche anglosaxonne et répond aux demandes immédiates des professionnels qui sont amenés à 
utiliser l'anglais médical en tant qu'outil de communication dans leur pratique quotidienne. Cette approche nous semble d'autant plus légitime que les professionnels de santé exerçant en France doivent communiquer en anglais, aussi bien à l'oral qu'à l'écrit, lors de congrès, dans le cadre de la mobilité internationale ainsi que dans le contexte contraignant des publications dans des revues en langue anglaise à comité de lecture et à facteur d'impact élevé1.

4 Ayant constaté que le discours spécialisé du domaine étudié par les enseignantschercheurs en anglais de spécialité entre parfois en conflit avec le discours effectif tenu ou attendu en milieu professionnel, nous avons porté une attention toute particulière aux ajustements nécessaires entre le discours effectif des professionnels et le discours pour les professionnels. Par discours des professionnels, nous entendons la pratique sociolinguistique entre professionnels de santé anglophones ou non anglophones utilisant l'anglais médical. Par discours didactisés pour les professionnels, nous entendons la pratique de l'anglais médical tel qu'il est enseigné par les enseignants d'anglais de spécialité aux futurs professionnels ou aux professionnels en formation continue dans un contexte non anglophone. Cette discordance est souvent liée à une connaissance imparfaite du milieu professionnel, d'où l'intérêt, si souvent préconisé, pour les enseignants-chercheurs de langue de consulter les acteurs du domaine afin de conduire une analyse des besoins langagiers susceptibles d'être recontextualisés et adaptés à l'enseignement de la langue spécialisée.

5 L'enseignement collaboratif (team teaching) existe depuis les années 1970 dans l'environnement pédagogique anglophone et, depuis, des recherches ont été développées à ce sujet dans le domaine universitaire (Winn \& Messenheimer-Young 1995 ; Michaelsen \& Richards 2005). Plus récemment, Séverine Wozniak a mis en avant la nécessité de prendre en compte l'acteur du domaine en tant que partenaire dans la démarche de recherche :

Le savoir du chercheur, comme celui de l'acteur, est fondamental, complémentaire dans le processus de recherche et déterminant pour la qualité des résultats dégagés. La perspective adoptée est beaucoup plus imbriquée : le chercheur est observateur, mais aussi partie prenante, l'acteur n'est pas seulement «l'objet » observé, mais il participe à la démarche de recherche. (2012: 27)

6 C'est dans cet esprit que nous nous sommes appuyés pour certaines actions sur divers travaux didactiques portant sur la recherche-action. Cette forme de recherche participante permet d'associer l'observation scientifique et l'expérience des acteurs de terrain, comme l'indique Jean-Paul Narcy-Combes :

La première étape relève du terrain : un problème y est identifié. Pour le résoudre, une réflexion débute: prise de recul, regard vers la (les) théorie(s) et/ou vers d'autres pratiques afin de trouver de nouvelles solutions. $(2005: 114)$

7 Srikant Sarangi (2015) insiste sur le concept de "communautés d'intérêt »" dont le caractère critique et transdisciplinaire permet d'atténuer le biais d'interprétation inhérent à toute situation d'observation et d'analyse. Dans ce contexte, notre participation à un projet européen nous a permis de côtoyer de nombreux acteurs intervenant dans des domaines très différents - des professionnels de santé, des biologistes, des didacticiens de l'anglais médical, des spécialistes de la linguistique appliquée, des juristes de différentes nationalités, etc. -, ce qui nous a permis d'intégrer leur point de vue à nos conclusions.

Dans cette étude, nous soulignons l'intérêt du travail collaboratif avec les professionnels de santé, les experts scientifiques et les linguistes associés à la 
communauté spécialisée. Afin d'illustrer cette synergie positive, nous décrivons et analysons quatre actions menées en collaboration étroite avec les professionnels de santé pour lesquelles une analyse des besoins langagiers a été nécessaire :

- un travail en binôme impliquant un professionnel de santé aguerri et un enseignant d'anglais médical lors de séances d'apprentissage du raisonnement clinique (ARC) à l'UFR Sciences de Santé de Dijon (depuis 1997);

- un projet de recherche-action (Charpy 2011) portant sur la relation entre représentation du réel et réalité professionnelle dans les romans de la fiction à substrat professionnel (FASP) et ayant fait appel au jugement critique d'une dizaine de médecins lors de séances de formation continue (année universitaire 2009-2010),

- un projet européen de certification en anglais médical appelé sTANDEM (standardised language examination for medical purposes), reposant sur l'expertise de professionnels de la santé, de la linguistique appliquée et d'enseignants d'anglais médical (Charpy \& Carnet 2014),

- la rédaction, à la suite d'un travail collaboratif avec plus de trente spécialistes médicaux, d'un ouvrage spécialisé sur l'anglais médical : L'anglais des spécialités médicales (Carnet \& Charpy 2014).

$9 \quad$ Nous présentons les divers apports des spécialistes médicaux lors de la mise en place et de l'aboutissement de ces projets collaboratifs. Prenant en compte les enseignements majoritairement bénéfiques des théories de l'enseignement collaboratif et de nos propres expériences, nous postulons qu'il est nécessaire d'intégrer les spécialistes anglophones ou ayant une bonne maîtrise de l'anglais médical à l'équipe des enseignants-chercheurs et des enseignants des UFR Sciences de Santé.

\section{L'apprentissage du raisonnement clinique en anglais : l'anglais des professionnels au service de l'anglais pour les futurs professionnels}

Depuis 1997, une des particularités de l'UFR Sciences de Santé de Dijon repose sur l'enseignement intégré, à savoir l'enseignement de la médecine en anglais en collaboration avec un professionnel de santé. La langue spécialisée est enseignée tout au long des six années des études médicales. Les trois premières années, est abordé l'anglais pour la médecine : principalement le lexique médical de base, les opérations de détermination et les structures grammaticales particulières propres à l'anglais médical (Carnet 1996). Progressivement, lors des trois dernières années, les étudiants sont formés à la pratique de la médecine en anglais ${ }^{3}$. C'est dans ce contexte clinique qu'ont été mises au point des séances d'ARC en anglais en quatrième et cinquième années des études médicales.

\subsection{Historique de l'apprentissage du raisonnement clinique à l'UFR Sciences de Santé}

11 Ces séances d'apprentissage sont nées d'une réforme pédagogique centrée sur l'étudiant et axée sur l'apprentissage contextualisé visant essentiellement les savoirfaire professionnels, qui est pratiqué à la faculté de médecine de l'université 
canadienne de Sherbrooke depuis 1987 (Chamberland 1998). Il est la base même des études médicales, et Anne Demeester et al. mettent en relief la difficulté de l'enseigner :

Le raisonnement clinique $(\mathrm{RC})$ se situe au cœur de la pratique médicale et soignante. Il s'exprime au quotidien par la capacité des praticiens à résoudre les problèmes cliniques posés par les patients. Former au RC, activité mentale peu observable et par essence peu accessible, est une mission difficile pour les enseignants cliniciens des filières de formation en santé. (2012:45)

Le raisonnement clinique est l'activité intellectuelle accomplie par le clinicien qui permet l'identification, l'anticipation et la collecte d'informations à partir d'une situation clinique particulière : elle intègre ces éléments aux connaissances et aux expériences antérieures et les utilise pour arriver à un diagnostic et à une prise en charge du patient appropriés. Cette approche repose sur deux prérequis essentiels, à savoir un bagage de connaissances spécifiques adéquat (à la fois médicales et linguistiques) et l'utilisation de stratégies de raisonnement clinique appelées également " processus analytique » (Nendaz et al. 2005).

Le raisonnement clinique se définit donc comme un ensemble de processus de pensée et de prises de décisions qui permettent au clinicien d'entreprendre les actions les plus appropriées dans un contexte spécifique de résolution de problèmes de santé (Higgs \& Jones 2008).

\subsection{Les séances d'ARC en anglais à l'UFR Sciences de Santé de Dijon}

14 C'est dans le contexte de l'enseignement intégré qu'il a été décidé, en 1997, d'assurer des séances d'ARC en anglais à Dijon. L'objectif principal était d'ajuster cette technique d'apprentissage à l'enseignement de l'anglais médical.

15 Ces séances d'une heure reposent sur le principe du jeu de rôles introduit dans les années 1970 dans le cadre de l'approche communicative interactive centrée sur l'apprenant et adapté ici au domaine de la médecine : l'un des étudiants joue le rôle d'un patient à qui a été donné un descriptif de signes et de symptômes ainsi que les principales constatations de l'examen clinique, sans bien sûr préciser la pathologie dont il souffre. Il peut donc répondre aux questions, mais ne possède pas tous les éléments. Les autres étudiants adoptent la posture du médecin et posent des questions au patient pour cerner les symptômes, tenter d'identifier la maladie à l'aide d'examens complémentaires et finalement proposer un diagnostic définitif et une prise en charge thérapeutique.

16 L'originalité de cet enseignement réside dans le fait que deux enseignants présents dans la salle travaillent en étroite collaboration : un médecin hospitalo-universitaire pour valider la démarche médicale inhérente au cas clinique, et un enseignant du département d'anglais médical pour valider la correction linguistique des échanges.

Chaque séance d'ARC repose sur un cas réel rencontré par le médecin dans sa vie professionnelle. À titre d'exemple, un médecin des urgences nous a proposé le cas d'une patiente souffrant de douleurs au niveau de l'estomac et dont le diagnostic final permettant de la sauver était un infarctus inférieur du myocarde. Tous les cas sont préparés en amont conjointement par l'hospitalo-universitaire et l'enseignant d'anglais afin de cerner les éléments lexicaux et syntaxiques utiles au bon déroulement de la 
consultation. Ces éléments sont intégrés pendant la séance afin qu'ils puissent être réutilisés en aval par les étudiants dans leur future pratique professionnelle.

Les médecins, qu'ils soient généralistes ou spécialistes, interviennent bénévolement ou dans le cadre de leur enseignement statutaire universitaire ${ }^{4}$. Dans les deux cas, ils sont intéressés, pour leur part, par la possibilité de pratiquer l'anglais dans des situations professionnelles avec un enseignant d'anglais. Ils sont tous issus de la formation médicale continue et ont obtenu leur diplôme inter-universitaire (DIU) ${ }^{5}$ d'anglais pour la médecine (Carnet et al. 1999), garant de leur bon niveau en anglais médical.

En début de séance, deux secrétaires sont désignés : l'un prend des notes au tableau ou sur ordinateur avec vidéoprojection pour que l'ensemble des étudiants ait une trace immédiate de ce qui est dit, et l'autre est chargé de faire une synthèse écrite plus détaillée de l'étude de cas qui sera distribuée aux étudiants lors de la séance suivante, après validation de l'enseignant d'anglais.

L'étape initiale de la séance d'ARC est l'interrogation du patient, qui permet de cerner les signes et symptômes actuels ainsi que les antécédents personnels, familiaux et sociaux du patient. À l'issue de cette première phase, un étudiant fait une synthèse orale grâce aux notes prises par le secrétaire de séance.

21 La deuxième phase porte sur l'examen clinique du patient. Bien sûr, les étudiants examinent le patient de manière virtuelle en posant les questions qui leur permettent d'obtenir les informations qu'ils auraient normalement en examinant physiquement le patient lors de la consultation. À nouveau, cette phase donne lieu à une synthèse orale faite par l'un des étudiants qui va reprendre tous les éléments importants obtenus grâce aux notes prises durant la séance.

22 La troisième phase porte sur le diagnostic différentiel (toutes les hypothèses qui sont évoquées en matière de diagnostic lors de l'étude d'un cas médical et dont une seule sera retenue). Les étudiants doivent formuler des hypothèses de diagnostic. Le rôle du professionnel de santé est à ce stade primordial, car les étudiants doivent justifier leurs hypothèses et les défendre de manière argumentée le cas échéant.

23 L'étape suivante consiste à confirmer ou à infirmer ces hypothèses. Les étudiants doivent prescrire les examens complémentaires pertinents en justifiant leur choix. Cette phase ultime conduit au diagnostic final et à la prise en charge du patient avec l'aval du médecin présent.

24 L'intérêt principal du travail avec le professionnel de santé est de recadrer constamment les étudiants dans le domaine de la méthodologie. En effet, lors de l'interrogatoire du patient, les étudiants ont tendance à poser des questions erratiques de manière non raisonnée au lieu de suivre une démarche progressive et rigoureuse prenant en compte les symptômes et les antécédents du patient.

À titre d'exemple, en cas de douleur aiguë survenue brutalement au mollet d'un patient, accompagnée de picotements et de perte de sensation, le professionnel de santé attend des étudiants qu'ils centrent immédiatement leur interrogatoire sur les « six Ps»: pain, pallor, perishing cold, paralysis, paraesthesia, pulselessness. De la même manière, en cas de soupçon de maladie infectieuse, le médecin attendra des étudiants que leurs questions portent sur les points suivants : statut vaccinal, récents voyages à l'étranger, allergies connues, contact avec des animaux, mode de vie (alimentation, relations sexuelles), etc. 
Ce travail présente également un intérêt linguistique. À titre d'illustration, outre le fait que la question fermée Do you smoke? soit insuffisante, il convient d'affiner l'interrogatoire par des questions plus précises du type How many cigarettes a day? How long have you been smoking? Since when have you been smoking? etc. Le médecin attendra ensuite que les étudiants convertissent les réponses en paquet-année (pack-years) ${ }^{6}$.

Ces quelques exemples montrent comment l'exposition récurrente au discours maîtrisé du professionnel de santé conduit l'enseignant d'anglais médical à intégrer les données pertinentes pour se construire un capital de culture médicale et d'effectuer les ajustements nécessaires pour proposer un discours approprié aux futurs professionnels de santé.

\section{La FASP médicale à l'épreuve des professionnels de santé : le réel fictif au service du réel professionnel}

Un projet de recherche-action portant sur une période allant de décembre 2009 à avril 2010 a été lancé à l'UFR Sciences de Santé de Dijon afin d'observer les perspectives didactiques et les potentialités pédagogiques offertes par des documents fictionnels tels que les medical thrillers, généralement peu utilisés par les professionnels de santé dans un contexte pédagogique. Pour atteindre cet objectif, nous avons engagé une réflexion collective associant des experts du domaine spécialisé afin de mettre en parallèle la représentation du réel proposée dans les œuvres de fiction et le vécu professionnel des praticiens (Charpy 2011).

\subsection{Le genre de la FASP médicale en action}

9 Ce genre a été défini initialement par Michel Petit dans plusieurs articles fondateurs $(1999,2000,2004)$. Il regroupe des œuvres de fiction contemporaines, émanant du genre populaire plus ancien du thriller, dans lesquelles le discours, les pratiques et la culture de divers milieux professionnels et des communautés spécialisées afférentes sont mis en scène, généralement par des professionnels en exercice ou ayant récemment exercé (on parle alors de substrat professionnel), ou encore des experts scientifiques ou des journalistes spécialisés n'ayant pas une connaissance directe de la pratique professionnelle (auquel cas il est question d'adstrat professionnel).

Diverses facettes de ce genre protéiforme, telles que sa dynamique spéculaire et les caractéristiques de son lectorat, ont été étudiées par Shaeda Isani (2004, 2009, 2010). Plus récemment, l'intérêt épistémologique du genre dans le cadre théorique et méthodologique des langues-cultures spécialisées a été confirmé par Michel Van der Yeught :

FASP works often provide a highly accurate mimesis of domains and SLs. As they are generally produced by talented insiders, they evoke specialised communities and cultures in scrupulous detail. Authoritative introductions to specialised worlds are not always available to SL teachers and learners and they should not be lightly brushed aside. Yet, the foremost merit of FASP is probably that its enticing verisimilitude is based on the authors' thorough mastery of specialised encyclopaedias which are part of specialised domains. (2016:60)

Parmi les genres rattachés à la notion centrale de fiction à substrat professionnel, la FASP médicale a fait l'objet de divers travaux de recherche ayant précédé le projet de 
recherche-action; ils avaient pour but d'explorer la typologie du genre, ses relations avec le milieu professionnel de la santé et ses évolutions, en particulier son caractère hybride de plus en plus marqué (Charpy 2004, 2005, 2010). Toutefois, ces études étaient fondées sur un a priori théorique formulé par Michel Petit :

Il s'agit donc d'une représentation du milieu professionnel en action, tel qu'on pourrait le connaître de l'intérieur si l'on en faisait partie, présentant $a$ priori toutes les garanties d'authenticité tant en ce qui concerne la langue et le discours que la culture. $(2004: 10)$

Il s'agissait donc de vérifier la pertinence de cette hypothèse en adoptant à la fois une démarche fondée sur la recherche participante (Catroux 2002; Mesnier \& Missotte 2003; Narcy-Combes 2005) et une approche pragmatique visant à associer une observation scientifique rigoureuse à l'expérience des acteurs de terrain.

Suivant les pistes de réflexion existantes sur la didactisation de fictions à substrat professionnel (Hardy 2004; Genty 2010), il a été décidé de retenir comme critères principaux pour valider ou invalider l'utilisation pédagogique des documents fictionnels étudiés leur crédibilité professionnelle et discursive.

En effet, dans le domaine spécialisé de la médecine, les textes couramment utilisés sont des sources primaires comme l'article de recherche ou, dans un contexte clinique, l'étude de cas, qui peuvent légitimement être considérés comme des documents authentiques. En revanche, les textes issus de la FASP ou de toute œuvre de fiction n'ont pas a priori une finalité professionnelle. Bien que censés être le reflet fictif du réel professionnel, ces sources secondaires ont pu faire l'objet de diverses altérations liées à l'expérience professionnelle directe ou indirecte de l'auteur et aux biais induits par le genre populaire $\mathrm{du}$ thriller, comme le recours au sensationnalisme ou au sentimentalisme.

\subsection{Le projet de recherche-action et ses principaux enseignements}

Étant donné que nous formons de nombreux professionnels de santé lors de cours de formation continue (DIVAM/DIVAS), il nous a semblé naturel de nous tourner vers ces consultants scientifiques pour conduire une expérimentation à visée principalement pédagogique.

Les professionnels de santé ayant validé un des deux DIU mentionnés ci-dessus pouvaient, s'ils le souhaitaient, perfectionner leur anglais médical dans le cadre d'une formation complémentaire non diplômante intitulée POST-DIVAM/POST-DIVAS. À ce stade, les participants avaient atteint un niveau B2/C1 leur permettant de lire et d'étudier des textes plus diversifiés et pas exclusivement techniques.

C'est dans ce contexte qu'il a été proposé aux praticiens inscrits en 2009-2010 de faire ce travail critique atypique à partir d'extraits de romans à dominante médicale en lieu et place des documents authentiques qu'ils avaient l'habitude d'utiliser comme sources primaires dans leur pratique professionnelle.

38 Six extraits de medical thrillers ou de romans comportant une scène à dominante médicale (Tableau 1) ont été soumis à dix professionnels de santé expérimentés ${ }^{7}$ lors de six séances de travail d'une heure trente, dont une heure consacrée à l'exploitation d'un questionnaire ciblé.

Tableau 1. Extraits étudiés 


\begin{tabular}{|l|l|l|l|}
\hline Titre & Auteur & Références & Genre \\
\hline The Surgeon & Tess Gerritsen & 2001, p. 19-24 & medical thriller \\
\hline The Society & Michael Palmer & 2005, p. 28-30 & medical thriller \\
\hline Locked in & Michael Esposito & 2007, p. 113-117 & medical thriller \\
\hline Past Lives & Ken McLure & 2007, p. 23-25 & medical thriller \\
\hline The Demon in the Freezer & Richard Preston & 2002, p. 52-54 & scientific thriller \\
\hline Mischief & Ed McBain & 1994, p.176-178 & police procedural \\
\hline
\end{tabular}

Les extraits ont été proposés sans le nom de l'auteur ni le titre du roman. Après une première lecture rapide effectuée par l'enseignant, puis une deuxième lecture silencieuse ayant pour objet une compréhension plus détaillée du passage, les participants devaient informer un questionnaire en anglais (Charpy $2011: 81$ ). C'est à partir de ce questionnaire-type, élaboré de manière à prendre en compte les besoins et l'expertise des professionnels en formation continue, que les données ont été recueillies auprès des acteurs participant au projet.

Les questions visant à vérifier l'authenticité professionnelle et la crédibilité discursive des extraits étaient les suivantes :

- Cet extrait est-il pertinent ou non d'un point de vue médical ou scientifique ? Pourquoi ?

- Le passage est-il ancré dans la réalité professionnelle: en d'autres termes reflète-t-il le discours, les pratiques et/ou la culture de votre communauté médicale ou scientifique? Justifiez votre réponse.

- Selon vous, qui a écrit ce passage : un médecin, un ex-médecin, un expert, un journaliste, un profane ... ? ${ }^{8}$

- Si vous le jugez nécessaire, faites des observations sur la relation entre réel professionnel ou scientifique et réel fictif dans ce passage.

41 Une phase finale de synthèse permettait de visualiser de manière globale l'authenticité professionnelle, l'intérêt pédagogique et la représentation fictive du réel dans l'extrait étudié. Avant de rendre leur questionnaire à l'enseignant, les participants devaient évaluer le passage selon les modalités générales suivantes: sur une échelle de zéro à dix, où situez-vous cet extrait ? (Tableau 2) ${ }^{9}$

Tableau 2. Évaluation globale des extraits étudiés

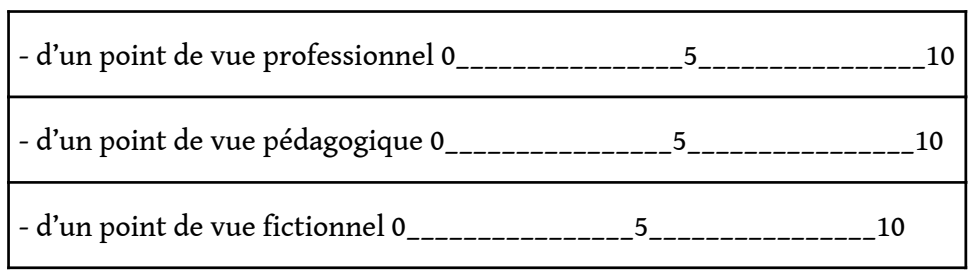

À l'issue de la phase d'appropriation et d'analyse critique des textes, un tour de table a permis aux participants de confronter et de discuter leurs points de vue respectifs quant à la pertinence et à l'authenticité professionnelle et discursive des extraits 
proposés en s'appuyant sur leurs réponses au questionnaire. Cette collecte de données a fourni à l'enseignant des indications précieuses en vue de la constitution d'une base de documents fictionnels suffisamment crédibles et motivants pour être utilisés en formation initiale ou continue. De la même manière, les termes médicaux ou scientifiques jugés utiles et validés par les professionnels lors de la discussion collective ont été collectés par l'enseignant afin de constituer une base lexicale de référence dans son enseignement initial.

Globalement, le lexique spécialisé de ces textes fictionnels a été perçu comme pertinent et généralement précis. Il convient de préciser que les professionnels de santé impliqués avaient une connaissance préalable relativement solide du lexique spécialisé en raison de leur participation antérieure à l'un ou l'autre des DIU médicaux proposés à Dijon. L'acquisition lexicale en interne a donc été minime et a porté principalement sur des termes à caractère plutôt spécialisé comme lead apron, the lesser omentum, instrument tray ou encore DIC (diffuse intravascular coagulation).

En revanche, les participants ont tous indiqué lors de la discussion qui a suivi que, en raison de la richesse du lexique spécialisé de base disponible dans les extraits, l'intérêt pédagogique principal de la séance résidait dans l'acquisition lexicale. En effet, cette acquisition pourrait être très utile pour les étudiants en médecine en formation initiale à condition que les termes et les situations cliniques soient adaptés à leur niveau de connaissances professionnelles, à savoir à partir de la troisième année des études de médecine.

Un autre intérêt pédagogique concerne l'évaluation du degré d'authenticité des situations professionnelles fictives. Les participants ont conclu que les pathologies et les procédures médicales ou les interventions chirurgicales étaient décrites avec précision et exactitude dans trois des six extraits (The Surgeon, The Demon in the Freezer et Locked in). Toutefois, ils ont estimé que quelques scènes manquaient de réalisme en raison de grossières exagérations portant sur certaines pratiques professionnelles comme l'ajustement manuel du masque chirurgical effectué dans le champ stérile par le chirurgien de Past Lives ou le port par le chirurgien de The Society de chaussures de sport personnelles au bloc opératoire, ce qui constitue une faute grave.

Selon les participants, l'extrait de Locked in, écrit par le radiologue Michael Esposito, présentait la meilleure adéquation entre représentation du réel et réel professionnel, alors que le passage extrait de The Society, pourtant écrit par Michael Palmer, un exurgentiste, leur semblait être le moins pertinent pour une exploitation pédagogique en raison du biais introduit par une quête systématique du sensationnalisme.

La discussion collective a également permis de mettre en exergue certaines différences culturelles entre communautés spécialisées. Ainsi, le passage extrait de The Surgeon correspond à une situation chirurgicale réaliste dans l'ensemble, mais le fait qu'une laparotomie soit effectuée directement dans le service des urgences, en dehors du bloc opératoire, entre en conflit avec la réalité professionnelle française. Il s'agit toutefois d'une simple différence de culture médicale, par ailleurs fort enrichissante d'un point de vue pédagogique, car de telles interventions ont bel et bien lieu aux États-Unis si la situation d'urgence l'impose.

Bien que les résultats de ce projet de travail collaboratif doivent être relativisés, en raison du petit nombre d'experts impliqués et du fait que, parmi eux, aucun n'était locuteur natif (même si la majorité avait effectué un stage de mobilité en pays 
anglophone), la démarche reste néanmoins pertinente. En effet, si l'essentiel de l'enseignement initial de l'anglais médical repose sur des sources primaires ayant reçu le sceau de l'authenticité scientifique ou des sources secondaires didactisées, notre étude tend à montrer qu'il est possible - sous condition de validation par des professionnels expérimentés - d'organiser certaines tâches à partir de sources secondaires comme des extraits de medical thrillers ou de thrillers comportant une scène à dominante médicale.

Ces textes, considérés légitimement comme appartenant à la fiction populaire, peuvent recéler des passages à fort potentiel professionnel, culturel et encyclopédique. Ces conclusions justifient une collaboration étroite entre l'enseignant d'anglais médical, dont le rôle est de proposer une approche raisonnée du discours et de la culture spécialisés pour les futurs professionnels, et les acteurs du domaine médical qui, grâce à leur expérience et à leurs compétences, connaissent intimement le discours et la culture spécialisés des professionnels.

\section{Le projet européen de certification en anglais médical sTANDEM : la communauté d'intérêt au service de la communauté de discours et de pratique}

50 Aujourd'hui, la certification en langues à vocation internationale ou universitaire repose principalement sur des tests internationalement reconnus comme le TOEFL (Test Of English as a Foreign Language), le TOEIC (Test of English for International Communication), mais aussi le CLES (certificat de Compétences en Langues de l'Enseignement Supérieur) en France.

51 Ces tests - à l'exception du GMAT (Graduate Management Admission Test) en anglais des affaires, de l'ILEC ${ }^{10}$ (International Legal English Certificate) en anglais juridique et de l'ICFE (International Certificate in Financial English) en anglais de la finance - couvrent une aire linguistique et culturelle de type généraliste permettant de valider des compétences du même ordre dans des situations de communication qui ne sont pas exclusivement professionnelles.

52 La plupart sont étroitement liés au CECRL (Cadre Européen Commun de Référence pour les Langues) (Bruderman et al. 2012), dont les descripteurs normatifs, cependant, ne sont pas toujours adaptés aux objectifs spécifiques des langues spécialisées, comme l'a fait remarquer Marie-Hélène Fries-Verdeil (2009).

53 En pratique, cependant, la mise en cohérence de la mosaïque d'objectifs spécifiques qui caractérise l'anglais de spécialité avec la multitude des descripteurs du CECRL n'est pas chose facile, les descripteurs se révélant souvent soit inexistants, soit inadaptés (2009: 118).

La certification en anglais médical - à l'exception de quelques tests de niveau CLES 3 développés à Toulouse Purpan, du test hongrois PROFEX (PROFiciency EXamination in languages for specific purposes), et du test australien OET (Occupational English Test) - reste largement inexplorée en dépit de la demande croissante des professionnels de santé. 


\subsection{Un projet collaboratif international en trois phases}

Devant l'absence de certifications reconnues internationalement par les professionnels de santé, un projet de quatrième génération (Tardieu 2013) reposant sur les besoins socio-professionnels des apprenants, financé par l'Union Européenne à hauteur de $398000 €$ a été conduit entre novembre 2011 et février 2015.

Le but de ce projet, piloté par un consortium d'enseignants-chercheurs européens (Autriche, France, Hongrie, Pologne, Roumanie, Royaume-Uni) et d'experts internationaux (Hollande, Japon, Malte), était de créer une certification internationale des compétences linguistiques mobilisables à des fins médicales pour les étudiants et les professionnels de santé non natifs dans les domaines de la médicine, de la pharmacie et des soins infirmiers.

Lors des diverses étapes de validation du projet, les partenaires ont adopté une approche interdisciplinaire associant linguistes, scientifiques et professionnels de santé afin d'éviter les biais induits par les points de vue exclusifs de chaque communauté et d'atteindre une interprétation collaborative. Cette démarche est en parfait accord avec S. Sarangi :

'Communities of interest' concern scenarios in which one crosses different communities of discourse/interpretative practice - where one may share 'interest' but not 'practices', 'discourses' and 'interpretations'. (2015 : 29)

58 Le projet s'est déroulé selon trois phases distinctes. La première a porté sur une analyse détaillée des besoins langagiers des professionnels de santé. Dans toute l'Europe, plus de 300 professionnels de santé, étudiants en santé et enseignants d'anglais médical ont complété trois questionnaires différents ${ }^{11}$. Les résultats de cette analyse ont conduit à la rédaction d'un manuel pour les concepteurs des futurs tests, reposant sur des situations de communication professionnelle authentiques, ainsi qu'à l'élaboration de descripteurs et de spécifications se démarquant du contexte normatif des certifications de type généraliste. Ainsi, tous les descripteurs du CECRL ont été réécrits afin d'adhérer aux besoins prioritaires des professionnels de santé.

Au cours de la deuxième étape, vingt-quatre séries de sujets ont été conçues par les partenaires travaillant en tandem sur des compétences bien précises : compréhension de l'écrit (Roumanie et Royaume-Uni), expression écrite (Autriche et Pologne), compréhension de l'oral (Autriche et Pologne) et expression orale (Roumanie et Royaume-Uni) ${ }^{12}$. Quatre séries de sujets ont été élaborées au niveau B1, dix au niveau B2, et dix au niveau C1. En accord avec les directives de la Commission européenne, dixneuf séries de tests ont été produites en anglais, et cinq en d'autres langues européennes (français, allemand, hongrois, polonais et roumain). Les tests élaborés par les tandems ont ensuite été soumis à un comité de validation multi-professionnel piloté à Dijon. Après cette phase de validation, les sujets ont été formatés, corrigés et finalisés.

L'étape finale a été une phase de mise en place de séances d'examen durant laquelle deux manuels ont été rédigés, l'un pour les examinateurs (2013) et l'autre pour les candidats (2014). Des examinateurs ont été formés et des tests ont été organisés simultanément dans plusieurs universités européennes en $2014^{13}$. Ces tests ont reçu un accueil favorable de la part des candidats, étudiants et professionnels de santé, qui, d'après un sondage effectué après les tests, les ont trouvés en adéquation avec leurs besoins. 
61 Grâce au site internet de sTANDEM $^{14}$, les objectifs du projet ont été largement diffusés et un grand nombre d'institutions liées au domaine spécialisé ont offert soutien et assistance. Parmi elles, on peut noter the Centers for Disease Control and Prevention (CDC), the Johns Hopkins School of Medicine, Myeloma UK, the National Institute of Health (NIH), the New England Journal of Medicine (NEJM), ainsi que the World Health Organization (WHO).

62 De même, un certain nombre de partenaires ont accepté de contribuer à la dissémination et à la promotion du projet. Parmi eux, le GERAS (Groupe d'étude et de recherche en anglais de spécialité) en France, MedPharm Jobs en Pologne, International Medical Publications en République Tchèque, the Iaonnina Medical School en Grèce, et the Virtual Medical Center en Australie.

\subsection{La collaboration entre professionnels de santé et enseignants d'anglais médical}

63 Comme indiqué ci-dessus, les descripteurs du CECRL ${ }^{15}$ sont apparus très rapidement trop généralistes pour les besoins langagiers des professionnels de santé. À titre d'illustration, un descripteur spécifique du niveau B1 qui concerne le domaine médical est un indicateur des limites de cette approche communicative généraliste: "I can provide concrete information required in an interview/consultation (e.g. describe symptoms to a doctor), but with limited precision ». Dans cet exemple, il est clair que le locuteur visé est le patient et non le professionnel de santé. C'est le domaine grand public qui est privilégié, et le domaine spécialisé n'est qu'évoqué.

64 C'est pourquoi, depuis le tout début du projet, les partenaires ont décidé de réécrire collégialement certains des descripteurs du CECRL afin de les adapter au mieux aux besoins et aux attentes des professionnels de santé. Ceci a pu être réalisé grâce à la présence dans notre groupe de travail d'un médecin odontologiste anglophone qui nous a constamment prodigué de précieux conseils sur les besoins et les attentes des professionnels de santé. Voici quelques descripteurs ${ }^{16}$ développés pour sTANDEM au niveau B1.

Can understand factual information in familiar professional topics (Listening Comprehension Skills)

Can understand important information in familiar professional topics (Reading Comprehension Skills)

Can make short announcements concerning everyday professional topics (Speaking Skills)

Can write short accounts of factual information in standard formats related to a specific genre (Writing Skills)

Lors des séances de validation des sujets, un travail collaboratif systématique a été fait pour chaque compétence ciblée. Pour ce qui est de la compréhension orale, la volonté de modéliser la réalité professionnelle a fait l'objet du choix de documents authentiques centrés sur le domaine spécialisé (patient information, health reports, undergraduate lectures au niveau B1; interviews with healthcare professionals, patients, and caregivers, medical news reports, case reports, graduate lectures au niveau B2 ; postgraduate and CME lectures/talks, research reports, conference presentations, case studies au niveau C1).

De la même manière, les genres associés à la compréhension de l'écrit ont été adossés à des supports liés à la pratique professionnelle (textbook extracts, product descriptions, information leaflets au niveau B1; official letters on professional topics, specialised case 
reports, referral letters, extended abstracts, application letters au niveau B2 ; research articles, letters to the editor, ethical approvals, editorials, informed consent statements, reading peer reviewers' comments au niveau $\mathrm{C} 1$ ).

En ce qui concerne la production écrite, une première tâche a porté sur des genres spécialisés ciblés (job applications, invitations, congress applications au niveau B1; cover letters, referral letters, complaint letters au niveau B2 ; letters to the editor, case reports, answering reviewers' comments au niveau $\mathrm{C} 1$ ). Une deuxième tâche a consisté à analyser un diagramme en donnant des informations factuelles, des interprétations et des arguments (niveau C1).

Pour tester l'expression orale, trois tâches ont été conçues, fondées sur l'interaction dyadique ou la production orale monologique : un court entretien concernant le métier et/ou le domaine de recherche du candidat, une simulation de conversation médecinpatient portant principalement sur l'interrogatoire du patient et une mini présentation sur un sujet professionnel à partir d'un diagramme ou d'un tableau.

69 L'un des intérêts majeurs de ce projet collaboratif a résidé dans le fait que tous les sujets conçus par les tandems ont fait l'objet de séances de validation bimensuelles organisées à Dijon. Chaque séance était conduite en liaison Skype avec les auteurs des maquettes de tests proposés et un panel d'experts composé de plusieurs membres provenant de la communauté d'intérêt: deux enseignants-chercheurs en langue appliquée, deux locuteurs natifs spécialistes d'anglais médical (britannique et canadien), et des médecins et/ou scientifiques ${ }^{17}$. Tous avaient un niveau $\mathrm{C} 1$ en anglais validé à l'issue de la formation continue. Compte tenu de la complexité scientifique et du degré de spécialisation médicale du projet, seule cette étroite collaboration entre linguistes et professionnels de santé européens nous a permis de le mener à son terme.

\section{L'anglais des spécialistes médicaux au service des professionnels de santé : un exemple de travail collaboratif}

70 La pratique active de l'anglais intéresse toutes les professions de santé, car elle est indispensable dans le contexte mondial actuel des échanges professionnels. Ceci est d'autant plus pertinent que les infirmiers/infirmières et les maïeuticiens/ maïeuticiennes ont obtenu récemment le statut universitaire et sont susceptibles d'avoir à effectuer des travaux de recherche au cours de leur carrière.

71 Par ailleurs, le professionnel de santé exerçant en France doit parfois accueillir un patient étranger non francophone dans son service à l'hôpital ou dans son cabinet médical; c'est le cas, en particulier, des médecins et des infirmiers/infirmières travaillant au service des urgences.

Il se peut également, comme c'est le cas pour la grande majorité des hospitalouniversitaires, qu'il ait à écrire un article de recherche, à présenter un poster ou à faire une communication en anglais lors d'un congrès médical afin de faire avancer sa carrière universitaire.

Il est également intéressant de noter que les étudiants en médecine et les professionnels sont de plus en plus souvent amenés à accéder à des banques de données numériques spécialisées en anglais ou à communiquer avec des collègues, avec des 
patients ou des particuliers anglophones autour de thèmes médicaux par le biais de médias sociaux comme Skype, Twitter (Myers 2015) ou encore les blogs et les wikis (Myers 2010).

\subsection{De l'intérêt d'un travail collaboratif autour des spécialités médicales}

74 À ce jour, grâce à la formation initiale prodiguée dans la plupart des UFR Sciences de Santé, les jeunes professionnels de santé sont capables de maîtriser l'anglais médical de base. Toutefois, l'anglais des spécialités médicales peut leur poser problème en raison de sa spécificité et de l'hyperspécialisation de son lexique. Ainsi, pour un étudiant ou un interne n'ayant pas encore effectué de stage en neurologie, la complexité de la terminologie liée au système nerveux peut être source de confusion. De la même manière, en oncologie, l'entretien avec le patient peut s'avérer délicat pour un praticien non aguerri.

75 Afin de combler en partie les lacunes communicationnelles et terminologiques liées à la spécialisation dans le domaine de la santé, les auteurs ont tenté de répondre aux besoins propres à vingt-cinq spécialités médicales de manière à permettre aux externes, aux internes ${ }^{18}$ et aux professionnels de santé en général de se familiariser avec les subtilités de la langue médicale spécialisée.

76 Certains ouvrages destinés à l'enseignement de l'anglais médical à l'université (Upjohn et al. 2006) reposent sur des bases méthodologiques et fonctionnelles solides, misant avant tout non pas sur la spécialisation, mais sur l'autonomie des apprenants. D'autres ouvrages (Faure 2011), tournés vers le lexique des spécialités médicales et fondés presque exclusivement sur l'expérience personnelle de l'auteur acquise lors de ses années d'enseignement à l'UFR Sciences de Santé, ne font pas appel directement à celle des professionnels de santé.

77 En vue de la rédaction d'un ouvrage spécialisé, nous avons entrepris d'adopter une approche fondamentalement différente en travaillant en collaboration étroite avec les spécialistes du CHU de Dijon afin de mieux prendre en compte leurs besoins, leurs attentes et leurs connaissances spécialisées.

78 Ce rapprochement entre enseignants d'anglais médical et professionnels de santé ayant pour objectif une production collaborative est peu usuel dans le domaine de la santé, même si une compilation quasi encyclopédique comme KWiC-WeB Fachwortschatz Medizin Englisch (Friedbichler \& Friedbichler 2003) reflète le travail en symbiose entre les deux enseignants autrichiens d'anglais médical et les spécialistes médicaux avec lesquels ils travaillent.

79 L'anglais des spécialités médicales (Carnet \& Charpy 2015) est donc le fruit de la collaboration entre deux enseignants-chercheurs en anglais médical à l'UFR Sciences de Santé de Dijon et vingt-neuf hospitalo-universitaires du CHU de Dijon ainsi que trois médecins libéraux bourguignons. Ce travail de longue haleine a été rendu possible grâce à l'intégration des professionnels de santé bourguignons dans les productions $\mathrm{du}$ département d'anglais médical de l'UFR Sciences de Santé. 


\subsection{Ouvrage spécialisé : conception et rédaction collaborative} d'anglais spécialisé et spécialistes du domaine. Chacune des vingt-cinq spécialités médicales abordées ${ }^{19}$ dans l'ouvrage a fait l'objet de plusieurs entretiens avec un, parfois deux, spécialiste(s) du domaine. Il convient de noter que l'ouvrage ne prétend pas à l'exhaustivité. En raison de l'hyperspécialisation de certaines disciplines, les choix définitifs ont été arrêtés lors des divers entretiens après consentement mutuel concernant les besoins de fragmentation et les priorités ciblés par les experts de chaque spécialité et par les auteurs.

81 La structure générale du livre et le contenu ont été finalisés par les auteurs après validation par les divers experts et relecture par un locuteur natif, enseignant d'anglais médical. Le but principal était de définir les thèmes et les rubriques pouvant permettre aux étudiants et aux professionnels de santé d'acquérir des connaissances lexicales et des compétences communicationnelles utiles dans des situations spécialisées, telles que la communication médecin-patient, pour laquelle la maîtrise linguistique de l'anamnèse et de l'examen clinique est indispensable, et l'aide à la lecture ou à la rédaction de l'article de recherche, qui nécessite une connaissance précise des sigles et acronymes professionnels.

Dans une première rubrique, le lexique de l'anatomie est présenté sous forme de glossaire. Les auteurs avaient initialement prévu un chapitre général consacré à l'anatomie; après consultation avec les experts - tout en étant conscients que cela pouvait donner lieu à certaines redondances - ils ont néanmoins décidé d'inclure une rubrique anatomique dans chaque chapitre ${ }^{20}$ en raison des spécificités terminologiques fines propres aux spécialités médicales traitées. À titre d'illustration, les experts en chirurgie et en dermatologie ont estimé qu'une telle rubrique était indispensable dans leur discipline.

Ensuite, une courte section abordant le lexique spécialisé de la physiologie permet de cerner les termes utiles. Cette rubrique n'a pas été jugée utile par les experts de psychiatrie et de santé publique consultés, mais elle est pertinente pour les autres spécialités en raison des nombreuses variations existantes; les fonctions neurologiques, digestives et respiratoires, par exemple, ont peu de points communs et demandent une connaissance précise de la terminologie concernant la physiologie dans chaque spécialité.

84 Afin de faciliter la tâche des lecteurs, une rubrique plus conséquente regroupe les mots-clés de la spécialité médicale, généralement classés en plusieurs sous-rubriques (généralités, signes et symptômes, maladies, tests et examens complémentaires, traitements ...). À titre d'exemple, une illustration concerne les subtilités offertes par les différents niveaux de langue utilisés pour rendre la notion de (urinary) urgency en français: là où deux urologues utiliseront le terme hautement spécialisé d'« urgenterie », un spécialiste s'adressant à un généraliste choisira sans doute le terme médical plus classique de "miction impérieuse ", alors que le généraliste parlera à son patient d'« urgence urinaire », voire d'« envie pressante».

La section suivante propose les principaux sigles et acronymes liés à la spécialité médicale. Tous les experts ont participé activement à cette rubrique particulièrement utile pour les étudiants ou les professionnels de santé souhaitant lire un article de recherche spécialisé, ou encore pour les chercheurs amenés à publier en anglais. Elle 
correspond à un degré de spécialisation élevé, comme le montrent les sigles suivants associés à l'imagerie médicale: BAS (barium swallow: «repas baryté») ou l'acronyme STEAM (stimulated echo acquisition mode : « mode d'acquisition par écho stimulé »).

La dernière rubrique $^{21}$ est davantage orientée vers la communication orale et l'interaction spécialisée entre médecins et patients; elle regroupe les expressions les plus courantes et les plus judicieuses utilisées lors de la consultation propre à chaque spécialité médicale (anamnèse et examen clinique). Ainsi, dans le domaine des maladies infectieuses, certaines questions délicates comme Have you had sex while under the influence of alcohol or drugs in the past year? How often do you use condoms with vaginal sex? With anal sex? ou Do you have sex with males only, females only or both? doivent-elles être posées avec tact. Les spécialistes d'oncologie et de gynécologie ont également apporté une contribution fine dans l'élaboration de cette rubrique car, dans leur spécialité, la communication avec les patients nécessite beaucoup de précautions oratoires.

Au terme de cette brève présentation de l'ouvrage, il convient d'ajouter que ce travail collaboratif a été rendu possible grâce à la proximité géographique du $\mathrm{CHU}$ et à la bienveillance des hospitalo-universitaires, facilitée par les liens établis de longue date entre eux et les enseignants du département d'anglais médical de l'UFR Sciences de Santé de Dijon. En effet, c'est cette relation harmonieuse reposant sur la confiance et la médiation des experts qui a permis de définir de nombreux paramètres pouvant être utiles aux professionnels de santé désireux d'approfondir leurs connaissances linguistiques des spécialités médicales.

\section{Conclusion}

À l'issue de cette présentation de quatre projets collaboratifs associant professionnels de santé, scientifiques, linguistes et enseignants en anglais médical à l'UFR Sciences de Santé de Dijon, nous souhaitons préciser que cette démarche pragmatique n'est pas exclusive, mais est complémentaire d'approches épistémologiques et méthodologiques qui visent à maîtriser la connaissance de la spécialité.

Néanmoins, dans le cadre institutionnel dans lequel nous exerçons, nous postulons que les enseignants de langue spécialisée doivent être intégrés à l'environnement professionnel de proximité. En effet, dans notre domaine spécialisé, ce milieu inclut le CHU, qui jouxte généralement l'UFR, ainsi que le réseau des médecins libéraux et des professionnels de santé qui travaillent dans un périmètre proche, à savoir la ville ou la région.

90 En raison de la proximité géographique entre l'hôpital et l'université, il semble naturel que les enseignants et enseignants-chercheurs d'anglais médical soient rattachés statutairement à l'UFR Sciences de Santé et non pas, comme c'est parfois le cas ailleurs, à un centre de langues transdisciplinaire. En effet, la position stratégique du département d'anglais médical au sein même de l'UFR permet de former les hospitalouniversitaires à l'anglais médical tout en bénéficiant de leur connaissance du discours spécialisé et de leur culture médicale. Grâce à ce contact permanent, il est possible de tisser des liens privilégiés avec le milieu professionnel afin de cerner les besoins prioritaires discursifs et communicationnels des étudiants et des professionnels de santé. 
91 Nous sommes tout à fait conscients que cette collaboration avec le milieu médical n'est possible qu'en raison du statut particulier des hospitalo-universitaires et que cette intégration peut s'avérer plus difficile dans d'autres UFR. Néanmoins, dans le contexte socio-professionnel qui est le nôtre, il est indispensable pour les enseignants de langue spécialisée de comprendre le discours et la culture des professionnels de santé par le biais d'actions collaboratives pour pouvoir les adapter et les recontextualiser afin de répondre aux besoins des (futurs) professionnels.

\section{BIBLIOGRAPHIE}

BRUdeRMAN, Cédric, Christine Demaison \& Faouzia BENDERDOUCHE. 2012. « Le CECRL : un outil pour construire une politique des langues? Retour d'expérience sur l'évaluation et la certification à l'université UPMC (2009-2011) ». Les Cahiers de l'APLIUT 3, 31-41.

CARNET, Didier. 1996. « Opérations de détermination caractéristiques de l'anglais médical ». Thèse de doctorat, Université de Bourgogne.

CARNET, Didier \& Jean-Pierre CHARPY. 2015. L'anglais des spécialités médicales. Paris : Ellipses.

CARNET, Didier, Sylvia MORGAN \& Félicie PASTORE. 1999. « Une idée novatrice en formation médicale continue : le D.I.V.A.M. (Diplôme Inter Universitaire d'Anglais pour la Médecine). Besoin superflu ou nécessité impérieuse ? ». La Presse médicale 27, 1474-1477.

CATROUX, Michèle. 2002. «Introduction à la recherche-action : modalités d'une démarche théorique centrée sur la pratique ». Les Cahiers de l'APLIUT 3, 9-19.

CHAMBERLAND, Martine. 1998. «Les séances d'apprentissage du raisonnement clinique (ARC) : un exemple d'activité pédagogique contextualisée adaptée aux stages cliniques en médecine ». Annales de médecine interne, 479-484.

CHARPY, Jean-Pierre. 2004. « Milieux professionnels et FASP médicale : de l'autre côté du miroir ». ASp 45-46, 61-79.

CHARPY, Jean-Pierre. 2005. « La FASP médicale et ses marges : textes de référence, prototextes et textes périphériques ». ASp 47-48, 83-101.

CHARPY, Jean-Pierre. 2010. «FASP médicale et substrat professionnel : le miroir éclaté ». ASp 57, 61-79.

CHARPY, Jean-Pierre. 2011. « Les premiers dictionnaires médicaux en langue anglaise : glissements diachroniques du spécialisé au non spécialisé ». ASp 59, 25-42.

CHARPY, Jean-Pierre \& Didier CARNET. 2014. « The European sTANDEM project for certification in medical English, Acceptability and transgression(s) ». ILCEA [en ligne], 19, mis en ligne le 27 juin 2014. <http://ilcea.revues.org/2475>.

DEMEESTER, Anne, Chantal EYMARD \& Dominique VANPEE. 2012. « Apprentissage du raisonnement clinique : difficultés identifiées en formation initiale sage-femme ", Revue française de pédagogie $181,43-54$. 
FAURE, Pascaline. 2010. «Des discours de la médecine multiples et variés à la langue médicale unique et universelle ». ASp 58, 73-86.

FAURE, Pascaline. 2011. Anglais médical [vocabulaire thématique, exercices corrigés]. Paris : Ellipses. FRIEDBICHLER, Ingrid \& Michael FRIEDBICHLER. 2003. KWiC-WeB Fachwortschatz Medizin Englisch. Stuttgart : Georg Thieme Verlag.

FRIES-VERDEIL, Marie-Hélène. 2009. « Mise en cohérence de l'anglais de spécialité et du CERCL en France : difficultés et enjeux ». ASp 56, 105-125.

GENTY, Stéphanie. 2010. « La validation du substrat professionnel dans La proie de Michael Crichton (Prey, US, 2002)». ILCEA 12, 1-12.

HARDY, Mireille. 2004. « Didactisation de fictions à substrat professionnel pour un enseignement de l'anglais du marketing : intérêt et faisabilité ». Mémoire de Master recherche sous la direction de Shaeda Isani, Université Bordeaux 2.

HIGGS, Joy \& Mark JONES. 2008. Clinical Reasoning in the Health Professions. Oxford : Butterworth Heinemann, Elsevier.

ISANI, Shaeda. 2004. « The FASP and the genres within the genre ». In PETIT, M. (dir.) en coll. avec S. ISANI, Aspects de la fiction à substrat professionnel. Collection travaux EA 2025. Bordeaux : Université Bordeaux 2, 25-36.

ISANI, Shaeda. 2009. « Specialised fictional narrative and lay readership: Bridging the accessibility gap ». ASp 56, 45-65.

ISANI, Shaeda. 2010. «Dynamique spéculaire de la fiction à substrat professionnel et didactique des langues de spécialité ». ASp 58, 105-123.

MESNIER, Pierre-Marie \& Philippe MISSOTTE. 2003. La recherche-action, une autre manière de chercher, de se former, transformer. Paris : L'Harmattan.

MICHAELSEN Larry \& Boyd RICHARDS. 2005. «Drawing conclusions from the learn-teaming literature in health sciences education: a commentary». Teaching and Learning in Medicine 17/1, 85-88.

MYERS, Greg. 2010. The Discourse of Blogs and Wikis. Londres : Continuum.

MYERS, Greg. 2015. « Social media and professional practice ». Insights into Medical Communication, Linguistic Insights 203. Berne : Peter Lang, 51-69.

NARCY-COMBES, Jean-Paul. 2005. Didactique des langues et TIC : vers une recherche-action responsable. Paris : Ophrys.

NENDAZ, Mathieu, Bernard CHARLIN, Vicki LEBLANC \& Georges BORDAGE. 2005. « Le raisonnement clinique : données issues de la recherche et implications pour l'enseignement ». Pédagogie Médicale 6, 234-253.

PETIT, Michel. 1999. « La fiction à substrat professionnel : une autre voie d'accès à l'anglais de spécialité ». ASp 23-26, 57-81.

PETIT, Michel. 2000. « Le paratexte dans la fiction à substrat professionnel ». Bulletin de la société de stylistique anglaise 21, 173-195.

PETIT, Michel. 2004. «Quelques réflexions sur la fiction à substrat professionnel : du général au particulier ». In PETIT, M. (dir.) en coll. avec S. ISANI, Aspects de la fiction à substrat professionnel. Collection travaux EA 2025. Bordeaux : Université Bordeaux 2, 3-23. 
RESCHE, Catherine. 2016. «Termes métaphoriques et métaphores constitutives de la théorie dans le domaine de l'économie : de la nécessité d'une veille métaphorique ». In FAsciolo, M. \& M. RossI (dir.), Métaphore et métaphores. Paris : Langue Française 189, 103-116.

SARANGI, Srikant. 2015. « Experts on experts: Sustaining 'communities of interest' ». In GOTTI, M., S. MACI \& M. SALA (dir.), Professional Discourse Studies. Insights into Medical Communication, Linguistic Insights 203, Berne : Peter Lang, 25-47.

TARDIEU, Claire. 2013. « Testing et certification ». RCDL 10, 237-251.

UPJOHN, Jonathan, Josiane HAY, Pierre-Emmanuel colle, Joëlle HIBBERT \& Amélie DEPIERRE. 2006. Minimum Competence in Medical English. Grenoble : EDP Sciences.

VAN DER YEUGHT, Michel. 2016. «A proposal to establish epistemological foundations for the study of specialised languages ». ASp 69, 41-63.

Why-sTANDEM-is-superior-to-general-academic-language-proficiency-testing < http:// www.standem.eu/wp-content/uploads/2013/01/Why-sTANDEM-is-superior-to-generalacademic-language-proficiency-testing.pdf>, consulté le 7 janvier 2014.

WINN Judith A. \& Trinka MESSENHEIMER-YOUNG. 1995. « Team teaching at the university level: What we have learned ». Teacher Education and Special Education 18/4, 223-229.

WOZNIAK, Sévérine. 2012. «L'angliciste de spécialité et son objet de recherche : contribution à la réflexion épistémologique ». ASp 61, 25-37.

\section{NOTES}

1. La carrière des hospitalo-universitaires est régie par le système des points SIGAPS (Système d'interrogation, de gestion et d'analyse des publications scientifiques). Le score des articles publiés est un indicateur qui tient compte à la fois de la catégorie de la revue et de la position des auteurs.

2. Ce concept sera approfondi (voir infra).

3. Contrairement aux autres UFR, les études médicales sont dérogatoires et comportent deux cycles de trois années chacun.

4. Le statut des hospitalo-universitaires leur impose de répondre aux besoins de la formation initiale à l'UFR Sciences de Santé, outre leur charge clinique et de recherche, sans avoir à effectuer un nombre d'heures prédéfini, contrairement aux enseignants-chercheurs du reste de l'université.

5. Deux diplômes inter-universitaires au contenu identique sont organisés par le département d'anglais de l'UFR Sciences de Santé de Dijon : le DIVAM (diplôme inter-universitaire d'anglais pour la médecine) en présentiel à l'intention des professionnels de santé de la région BourgogneFranche-Comté, et le DIVAS (diplôme inter-universitaire d'anglais pour la santé) en semiprésentiel et à distance pour les professionnels de santé de toute la France.

6. Pour information, l'expression 10 paquets années correspond à un 1 paquet par jour pendant 10 ans, ou 10 cigarettes par jour pendant 20 ans, ou 5 cigarettes par jour pendant 40 ans.

7. Un cardiologue, un dermatologue, trois chirurgiens, une anesthésiste, deux urgentistes, un psychologue du domaine sportif, une infirmière. La plupart, en tant qu'hospitalo-universitaires, avaient une expérience de l'enseignement à l'UFR Sciences de Santé.

8. Les réponses à cette question ont été vérifiées lors de la mise en commun des résultats. Les réponses des participants correspondaient majoritairement à la réalité de la profession ou de l'ex-profession des auteurs. 
9. À titre indicatif, l'intérêt professionnel de The Demon in the Freezer et de Locked in $(7,5)$ a été mis en avant alors que Past Lives (4) a été jugé peu réaliste. Les extraits de The Demon in the Freezer et de The Surgeon (7) ont présenté un intérêt pédagogique certain tandis que l'extrait de Past Lives $(4,5)$ n'a pas retenu l'attention des participants. D'un point de vue fictionnel, c'est le passage tiré de Locked in (8) qui a suscité le plus d'intérêt.

10. Ce test a pris fin en décembre 2016.

11. Les réponses aux questionnaires sont disponibles sur le lien suivant: < http:// www.standem.eu/wp-content/uploads/2012/04/Results-of-language-needs-analysis.pdf>.

12. La France avait pour responsabilité la correction et la validation des sujets.

13. Parmi lesquelles, Créteil et Angers pour la France, Innsbruck en Autriche, Pecs en Pologne, Targu Mures en Roumanie, Cracovie en Pologne..

14. sTANDEM site <http://www.standem.eu/>.

15. Council of Europe. Common European Framework of Reference for Languages 1, 177. <http:// www.coe.int/t/dg4/linguistic/cadre_en.asp>.

16. STANDEM descriptors < http://www.standem.eu/wp-content/uploads/2012/07/ sTANDEMdescriptors_03.08.201.pdf>.

17. Parmi eux, se sont succédé une gynécologue, une sage-femme, deux internistes, un chirurgien cardio-vasculaire, un dermatologue, deux biologistes, un anatomopathologiste et une spécialiste de la reproduction assistée.

18. D'une part les étudiants en médecine du $1^{\mathrm{er}}$ et du $2^{\mathrm{e}}$ cycle en stage d'observation, d'autre part, les étudiants du $3^{\mathrm{e}}$ cycle en stage de responsabilité à l'hôpital.

19. À savoir l'anatomopathologie, la cardiologie, la chirurgie et l'anesthésie, la dermatologie, l'endocrinologie, la gastro-entérologie, la gériatrie, la gynécologie et l'obstétrique, l'hématologie, l'imagerie médicale, les maladies infectieuses, la médecine d'urgence, la médecine générale, la médecine interne et l'immunologie, la neurologie, l'oncologie, l'ophtalmologie, l'otorhinolaryngologie, la pédiatrie, la pneumologie, la psychiatrie, la santé publique, la rééducation, la rhumatologie, l'urologie et la néphrologie.

20. À l'exception de ceux consacrés à la psychiatrie et à la santé publique, où cette rubrique n'était pas pertinente.

21. Cette section n'a pas été jugée utile en anatomopathologie et en santé publique.

\section{RÉSUMÉS}

D’un point de vue synchronique, le discours effectif tenu en milieu professionnel, fondé sur des besoins pragmatiques immédiats, entre parfois en conflit avec le discours spécialisé du domaine étudié par les enseignants-chercheurs. Ainsi, ceux-ci ont-ils tout intérêt à consulter les acteurs $\mathrm{du}$ domaine afin de cerner leurs besoins langagiers dans le cadre de projets collaboratifs. Cet article pose le problème des ajustements pertinents entre discours de professionnels et discours pour (futurs) professionnels dans la mise en place de quatre projets à visée pédagogique : des séances d'aide au raisonnement clinique en binôme, un projet de recherche-action sur la FASP, un projet européen de certification en anglais médical (sTANDEM) et un ouvrage spécialisé (L'anglais des spécialités médicales, Ellipses 2015). S'inspirant des travaux sur l'enseignement collaboratif et sur leur expérience personnelle, les auteurs postulent qu'il est crucial que les 
professionnels ayant une bonne maîtrise de l'anglais travaillent en étroite collaboration avec les enseignants d'anglais médical

From a synchronic viewpoint, the language and discourse actually observed in the medical workplace is based on immediate pragmatic needs, and sometimes clashes with the specialised discourse of the domain-specific area studied by language researchers. Consequently, it is in their best interest to join forces with the actors of the domain to target their language needs in the context of collaborative work. This article raises the question of the relevant adjustments between the actual discourse of healthcare professionals and the discourse taught to (future) professionals through four pedagogically-oriented projects: Clinical problem solving sessions involving two teachers, a medically-based fiction, action research project, a European project on language certification (sTANDEM), and a specialised publication. Based on previous research work on collaborative teaching and their own experience, the authors postulate that it is crucial for healthcare professionals with a good command of English to work hand in hand with teachers of medical English.

\section{INDEX}

Mots-clés : anglais médical, besoins, discours professionnel, pédagogie, recherche-action, travail collaboratif

Keywords : action research, collaborative work, language needs, medical English, professional discourse, second language acquisition

\section{AUTEURS}

\section{DIDIER CARNET}

Didier Carnet, agrégé d'anglais, est maître de conférences à l'UFR Sciences de Santé de Dijon. Il est membre de l'EA 4182 de l'Université de Bourgogne-Franche-Comté. Il est l'auteur du Lexique des termes médicaux, anglais-français et français-anglais (Ellipses 2006) et du Lexique anglais/français à l'usage des professions paramédicales (Ellipses 2010), ainsi que le co-auteur du guide pratique La communication orale scientifique en anglais (Ellipses 2002), de L'article de recherche scientifique en anglais (Ellipses 2010), de L'anglais des spécialités médicales (Ellipses 2015) et de L'anglais médical à la faculté (4e éd.) (Ellipses 2016). Sa recherche porte sur la linguistique, l'analyse du discours médical et la didactique en anglais médical. didier.carnet@u-bourgogne.fr

\section{JEAN-PIERRE CHARPY}

Jean-Pierre Charpy, agrégé d'anglais, est maître de conférences à l'UFR Sciences de Santé de Dijon. Il est membre de l'EA 4182 de l'Université de Bourgogne-Franche-Comté. Il est le co-auteur du guide pratique La communication orale scientifique en anglais (Ellipses 2002), de L'article de recherche scientifique en anglais (Ellipses 2010), de L'anglais des spécialités médicales (Ellipses 2015) et de L'anglais médical à la faculté (4e éd.) (Ellipses 2016). Ses domaines de recherche portent sur l'étude diachronique de l'anglais médical, la FASP médicale et le discours scientifique. jeanpierre.charpy@u-bourgogne.fr 